\title{
Éléments de panorama
}

\subsection{Au commencement était la mécanique}

Au commencement était la mécanique...*

L'origine de la mécanique remonte à des temps anciens mais son histoire ne commence véritablement qu'avec Galilée, qui en a posé les fondements. Depuis, la mécanique a traversé les époques, marquant de sa présence les grandes avancées technologiques et les révolutions de tous les secteurs de l'économie : les transports, la construction, la communication, la santé, l'énergie, etc.

Discrète par endroits, centrale ailleurs, la mécanique a toujours été l'un des vecteurs du progrès humain. Elle participe de toutes les industries, devenant de plus en plus difficile à isoler, à identifier...

\subsubsection{L'industrie au cœur de l'économie}

L'industrie regroupe l'ensemble des activités économiques qui produisent des biens matériels par la transformation et la mise en ouvre de matières premières. Au cours des deux derniers siècles, l'industrie a permis de produire trente fois plus en deux fois moins de temps. C'est le progrès* industriel qui a généré l'augmentation des richesses et l'amélioration des conditions et la réduction de la durée du travail. Dans notre pays, l'industrie se situe toujours au cœur de la création de richesses et de l'emploi puisqu'elle représente $85 \%$ des dépenses de R\&D et $80 \%$ des exportations de biens et de services, réalise 13,8 \% du PIB national et emploie un peu plus de $13 \%$ des actifs salariés. Se concentrant sur ses savoir-faire clés, elle a progressivement confié beaucoup d'activités périphériques au secteur des services à l'entreprise industrielle ; ceux-ci ont vu doubler leurs effectifs depuis le début des années 1980, gagnant près de deux millions d'emplois, pendant que la production manufacturière stricto sensu en perdait un million et demi. Néanmoins, sans socle industriel, une grande partie des emplois de services n'existerait pas. Les industries manufacturières sont ainsi présentes partout dans le monde et progressent fortement dans les pays émergents ou se maintiennent inexorablement,

* Max von Laue, Histoire de la physique, 1946. 
dans certaines économies hautement développées pour lesquelles elles constituent un «fer de lance » des exportations.

\subsubsection{La mécanique au cœur de l'industrie}

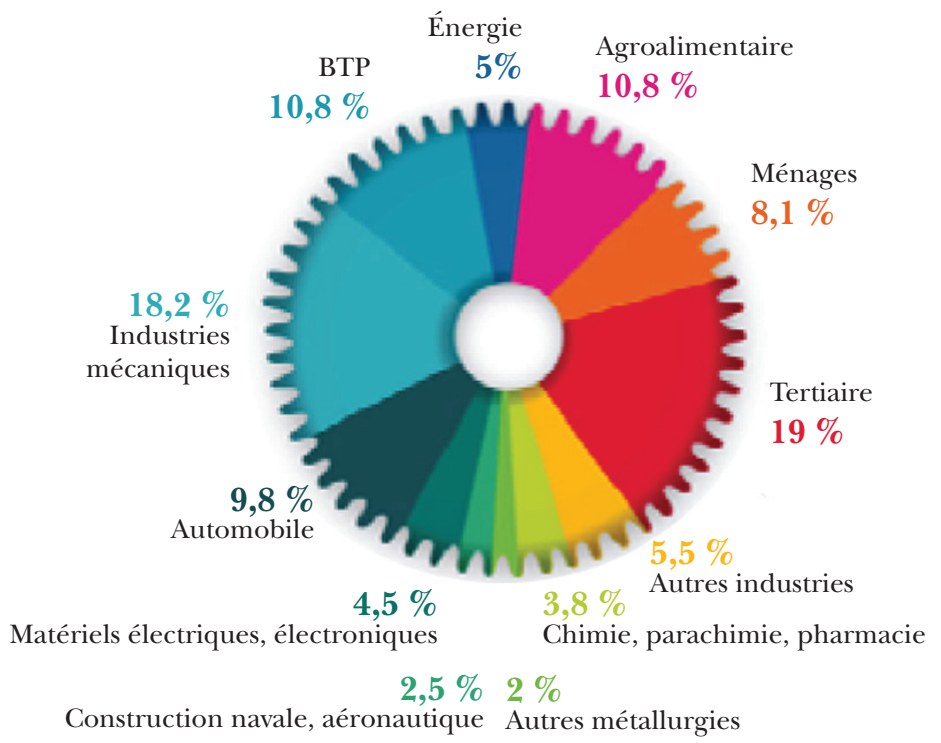

FigURE 1.1. - Les principaux secteurs économiques clients des industries mécaniques. Source : FIM.

Au cœur du développement de toutes les activités industrielles, conjuguant une panoplie toujours plus vaste de nouvelles technologies et de techniques plus traditionnelles, les industries mécaniques interviennent dans des métiers et sur des marchés extrêmement variés : l'automobile, l'aéronautique, l'énergie, le BTP, le transport, l'électronique, l'informatique, la construction navale, l'agroalimentaire, le textile, le médical, la chimie, ou encore la parachimie et la pharmacie... Là où il y a production industrielle, il y a mécanique : un moteur d'avion, un capteur, un autocuiseur, une paire de lunettes, un chariot élévateur, un robot, un extincteur, une fourchette, un scanner, une éolienne...

L'industrie mécanique travaille et assemble des matériaux qui vont bien audelà des matériaux métalliques. Elle intègre en permanence des technologies de toute nature, particulièrement celles de l'électronique et de l'informatique, afin de fournir des ensembles de plus en plus complexes, répondant à des fonctions habituelles ou innovantes. Au-delà, elle apporte des solutions pour assurer la qualité et réduire les prix des produits requis par le marché.

Omniprésente, transversale - des secteurs de haute technologie dans lesquels la France se positionne parmi les meilleurs, comme le spatial, l'aéronautique, l'énergie, le ferroviaire grande vitesse ne peuvent se développer sans le concours des scientifiques, ingénieurs et industriels de la mécanique -, celle-ci reste quasiment invisible, probablement parce que sa structure est très dispersée, constituée 
pour l'essentiel de moyennes et petites entreprises qui ont souvent choisi une stratégie de niche compatible avec des ressources financières limitées. La mécanique reste, en outre, un domaine très technique dont la relative faiblesse de rentabilité n'attire pas le monde financier.

Elle recouvre trois grands domaines d'activité : la transformation des métaux (sous-traitance, outillages, articles de ménages), les équipements industriels (machines, systèmes de production, composants) et la mécanique de précision (optique, instruments de mesure). La force de la mécanique en France ne réside pas seulement dans sa capacité à produire des solutions, mais aussi et surtout dans celle de les concevoir et de les rendre possibles. Cet indéniable atout résulte du savoir-faire de plus de 628000 femmes et hommes qui entretiennent et transmettent les compétences d'hier, et inventent celles de demain.

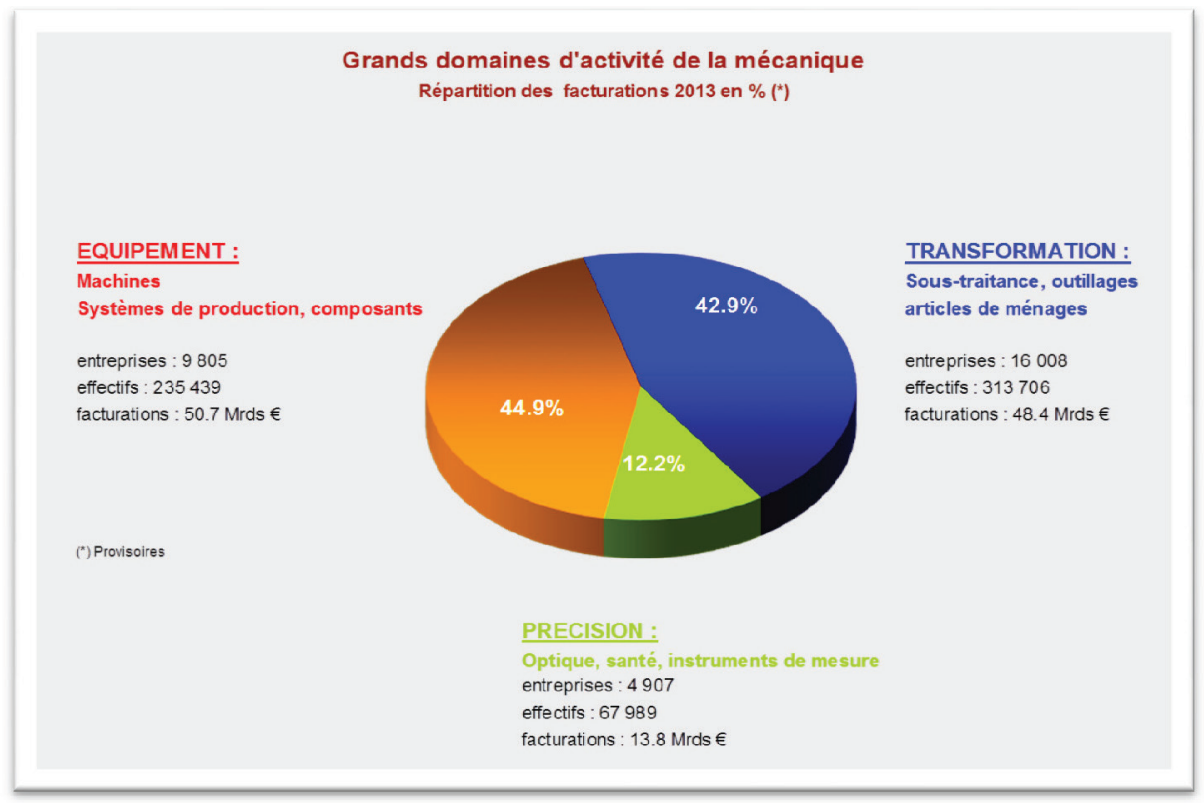

Figure 1.2. - Les trois grands domaines d'activités des industries mécaniques. Source : FIM.

\subsubsection{Un acteur économique majeur, un tissu de PME}

Dans un environnement industriel malmené, l'industrie mécanique française maintient son activité, avec près de 113 milliards d'euros de chiffre d'affaires (source FIM). Quelle que soit la taille de ses entreprises, la mécanique française participe au développement économique de la France et de ses régions, et pense son avenir au niveau international. 


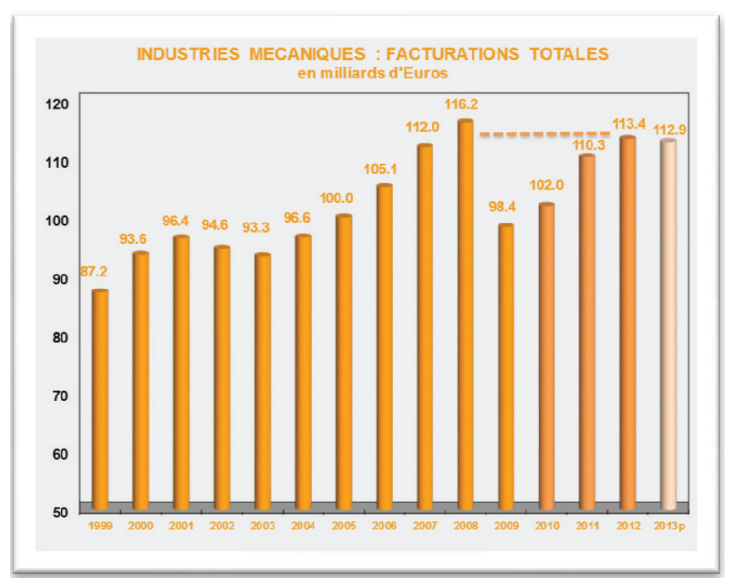

Sources : INSEE FIM - 30720 Entreprises de plus de un salarié

Figure 1.3. - Évolution du chiffre d'affaires des industries mécaniques.

L’industrie mécanique regroupe trente métiers, fédérés par la Fédération des Industries Mécaniques (FIM). En charge des intérêts économiques et techniques de ces trente professions, la FIM a pour objectif d'aider les mécaniciens à concevoir, produire et vendre en France et à se développer sur l'ensemble des marchés internationaux. Elle assure, sur les plans français et international, la défense et la promotion de ce secteur majeur de l'industrie française.

Accompagnant les entreprises dans leurs préoccupations collectives, la FIM leur apporte aussi des solutions dans tous les domaines de leur activité mécanicienne : juridique, environnement, marchés, technique, fiscalité, international, financement...

Le tissu des industries mécaniques est essentiellement composé de TPE et PME. $89 \%$ des entreprises de mécanique comptent de 10 à 100 salariés. Par rapport aux pays concurrents et, en particulier l'Allemagne, la France compte peu de moyennes entreprises entre 250 et 3000 salariés. En Allemagne, ces moyennes/ grosses entreprises sont deux fois plus nombreuses qu'en France dans la transformation des métaux et la précision, et trois fois plus pour les biens d'équipement (source : «2010-2020 : l'industrie au cour de la croissance durable », GFI). En quelques chiffres (source : INSEE - SESSI) :

- 43,7\% d'entreprises de 10 à 20 salariés,

- 33,2 \% d'entreprises de 21 à 50 salariés,

- $12 \%$ d'entreprises de 51 à 100 salariés,

- 9,4\% d'entreprises de 101 à 500 salariés,

- 1,7\% d'entreprises de plus de 500 salariés.

Ces entreprises sont majoritairement sous contrôle capitalistique français, avec des centres de décision en France. 


\subsubsection{Une réussite qui se joue à l'international}

Les mécaniciens sont depuis longtemps tournés vers l'international et près de la moitié de leur production est aujourd'hui exportée. Les PMI de la mécanique ont développé des stratégies pour partir à la conquête des marchés étrangers (innovation, alliances, travail collaboratif, qualité...). L'évolution du chiffre d'affaires et des effectifs est étroitement liée à la croissance des exportations. La conquête de nouveaux marchés (notamment hors de la zone euro) permet aussi aux entreprises de s'adapter à la demande mondiale et d'innover. Pour toutes ces raisons, l'avenir de l'industrie mécanique réside dans sa compétitivité et sa réussite à l'international.

Le marché communautaire constitue encore le principal débouché (55,3\% de notre production) et le principal fournisseur $(66,1 \%)$ de la France.

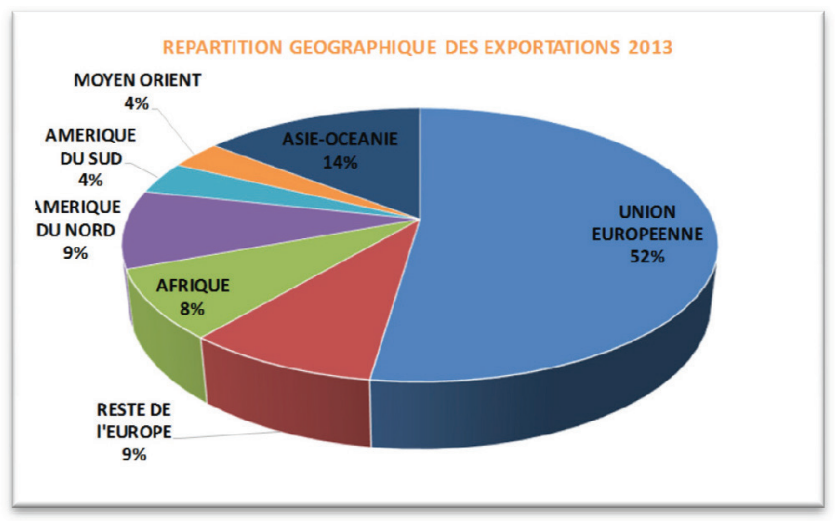

Figure 1.4. - Les débouchés à l'export des industries mécaniques.

Cet « eurotropisme " n'est pas propre à notre pays. En effet, schématiquement, les échanges des cinq premiers producteurs européens (Allemagne, Italie, France, Royaume-Uni et Espagne) s'effectuent majoritairement entre eux. Cependant, l'Allemagne et l'Italie couvrent respectivement leurs importations à hauteur de $177 \%$ et $200 \%$, tandis que la France est déficitaire. Relativement peu tournée vers l'exportation hors de l'Union Européenne, la France accuse un retard par rapport à ses principaux concurrents européens.

La France exporte principalement dans des zones à faible croissance et insuffisamment dans les zones de grand développement (Asie, Amérique latine). En 2013, les cinq principaux pays clients de la mécanique française sont l'Allemagne (14,8\% du total des exportations), les États-Unis (7,4\%), le Royaume-Uni (6\%), l'Espagne $(5,7 \%)$ et l'Italie $(5,6 \%)$.

\subsection{5. À la base des grandes innovations industrielles}

L'industrie mécanique est un acteur majeur en matière d'investissements de R\&D ; ils peuvent varier de 2 à $8 \%$ du chiffre d'affaires en fonction du secteur et de la 
taille des entreprises mécaniciennes. Les industries mécaniques représentent $35 \%$ des dépenses nationales de $\mathrm{R} \& \mathrm{D}$ des entreprises. Il existe des « champions » dans tous les domaines de la mécanique : les équipements et les process pour l'agroalimentaire, le BTP, la manutention, le textile, l'optique, le médical, les composants de transmission de puissance...

La mécanique française est avant tout un incroyable levier d'innovation pour tous les secteurs de l'économie. Son inventivité rend possible des avancées de premier plan, visibles à travers les réalisations de grandes entreprises : l'Airbus, l'AGV (le TGV nouvelle génération), le Grand Stade Lille, ITER... La recherche fondamentale, en physique notamment, nécessite elle aussi de recourir aux compétences de la mécanique. Les nombreuses filières industrielles clientes comptent sur son aptitude à innover, à créer, à inventer pour toujours offrir les meilleurs produits et les meilleures prestations que le marché peut attendre.

Pour bien servir ces filières clientes, l'industrie mécanique doit faire face à un certain nombre de défis économiques et techniques :

- la mondialisation des marchés et des capitaux ne maintient aujourd'hui sur le territoire français que des productions ou produits possédant un avantage concurrentiel certain, alors que les marchés régionaux et nationaux semiprotégés étaient courants dans les années 1980. Les conditions socio-économiques françaises nécessitent des efforts soutenus de productivité et surtout d'innovation pour maintenir le potentiel industriel ;

- la notion d'entreprise étendue dans les filières clientes de la mécanique se généralise. Cette démarche conduit à confier à l'entreprise fournisseur la responsabilité d'une solution technique complète, qu'il s'agisse du choix du matériau et de sa mise en œuvre, de la réalisation d'une fonction mécanique ou d'un équipement de production adapté au processus du client. La responsabilité de recherche et développement est reportée sur de plus petites entreprises élargissant leur rôle de sous-traitant de capacité ou de fabricant de composants ou d'équipements "sur étagère " à celui de fournisseur de solutions. Elle nécessite, de plus, l'intégration de l'entreprise mécanicienne dans le processus de conception du donneur d'ordre autour de modèles virtuels l'obligeant à des efforts importants d'évolution de ses méthodes de travail et d'informatisation. Par ailleurs, les attentes des clients d'offres de solutions complètes (offres de fonction globale au lieu d'offres de composant ou de sous-produit) rendent nécessaires l'association de compétences et donc le travail en réseau avec des entreprises ou organismes disposant de compétences complémentaires ;

- la rapidité de mise sur le marché des produits et leur personnalisation sont des facteurs désormais critiques. Cette évolution modifie en profondeur les processus de conception (conception simultanée) et de fabrication (procédés adaptés à des séries plus faibles, flexibilité des systèmes de production, diminution des temps de mise au point des outillages) ;

- les impératifs réglementaires sont croissants, qu'il s'agisse de bruits, de vibrations ou de pollution (économie d'énergie, limitation des émissions, cycle de vie des produits dont leur recyclage en fin de vie). Ils nécessitent des efforts soutenus de recherche et développement. Bien que souvent pénalisantes 
sur le plan économique, ces contraintes n'en constituent pas moins des opportunités de s'assurer d'un avantage concurrentiel par l'innovation, sous réserve que la réglementation et les modalités de son application soient bien homogènes dans les différents pays.

\subsubsection{Des outils technologiques indispensables}

Pour faire face à ces défis économiques modernes, les entreprises de la mécanique évoluent et innovent sans cesse. L'innovation, la capacité à réagir, à s'adapter aux nouvelles attentes du marché sont essentielles pour que l'industrie française renforce sa compétitivité et poursuive un développement durable.

Si l'innovation en tant que telle consiste en la création de nouveaux produits ou nouveaux procédés, celle-ci s'appuie, en général, sur des compétences, technologies, idées nouvelles qui peuvent provenir de veille technologique, d'actions de recherche et développement réalisées par d'autres entités que l'entreprise innovante elle-même. C'est le cas, entre autres, des laboratoires et centres de recherche...

Malgré la compétence des chercheurs, les PME éprouvent des difficultés à asseoir leurs développements sur des relations étroites et durables avec le monde de la recherche. Pour résoudre cette difficulté, l'organisation professionnelle mécanicienne, représentant un large tissu de petites et moyennes entreprises, conscientes de l'importance de l'innovation, a créé des Centres Techniques Industriels (CTI) pour disposer de moyens collectifs de recherche technologique, mettre en commun des moyens pour permettre aux entreprises de partager des équipements, des compétences et des informations qui, sans cela, leur seraient souvent demeurés inaccessibles. La FIM a ainsi créé le CETIM en 1965. Les principales missions du CETIM consistaient à apporter aux entreprises ressortissantes des moyens et des compétences pour accroître leur compétitivité, participer à la normalisation, faire le lien entre la recherche scientifique et l'industrie, promouvoir le progrès des techniques et aider à l'amélioration du rendement et à la garantie de la qualité.

Aujourd'hui, les technologies évoluent très rapidement. Les entreprises doivent s'adapter en permanence dans un monde de plus en plus globalisé. Le CETIM, au service des entreprises, met en œuvre des programmes de recherche technologique et organisationnelle en concertation avec les professions, dans un contexte de plus en plus ouvert au niveau européen et international. Les missions actualisées du centre technique se déclinent aujourd'hui autour de grands axes technologiques tels que :

- organiser un transfert et donc une appropriation par les entreprises indépendamment des résultats des recherches collectives pour leur permettre d'innover et de faire évoluer leurs métiers, leurs propres produits et services, et de développer le niveau de formation et de qualification de leurs salariés ;

- offrir l'accès aux PME aux grands programmes de recherche publique, nationaux et européens ou, à défaut, à leurs retombées technologiques ;

- soutenir la présence des PME dans la normalisation, outil stratégique de défense des intérêts économiques, en contribuant de manière significative à 
la logistique des bureaux de normalisation sectoriels, de manière à en faciliter l'accès aux experts des entreprises et en apportant un soutien technique aux commissions de normalisation à travers la participation d'experts des CTI aux travaux de normalisation et la réalisation d'études prénormatives le cas échéant ;

- organiser une veille stratégique des technologies et des marchés pour aider les entreprises à connaître les évolutions prévisibles des technologies et des marchés, et ainsi anticiper les ruptures (développement du travail en réseau, concept d'entreprise étendue, développement des services...), faire évoluer leurs produits afin qu'ils respectent les réglementations ;

- aider les entreprises à améliorer leurs performances industrielles et environnementales par l'évaluation et l'organisation de la conception, de la fabrication, de la maintenance et du contrôle (modélisation et simulation des procédés et des produits), à développer des composants et des équipements fiables, performants, compétitifs et respectueux des exigences réglementaires, à améliorer la productivité et la maîtrise de l'outil de production et à surveiller la conformité et la performance des produits.

D'autres outils sont à la disposition des entreprises.

Les pôles de compétitivité sont une opportunité ; ils constituent un outil important de politique industrielle pour renforcer la compétitivité de l'industrie française. Ils offrent une précieuse passerelle vers le monde de la recherche académique. Ils sont implantés sur les territoires, donc très proches des acteurs de terrain, et les entreprises peuvent orienter les thèmes de recherche pour qu'ils répondent aux besoins du marché. La coordination de leurs actions, comme le permet la plate-forme MECAFUTURE, portée par la FIM en partenariat avec le CETIM, qui fédère huit pôles de compétitivité dédiés à la mécanique et aux matériaux (Arve Industries, Elastopôle, EMC2, Matéralia, Microtechiques, Plastipolis, Pôle de l'industrie nucléaire, ViaMéca), est essentielle pour concentrer les efforts, éviter les redondances, consolider les projets de recherche et faire plus au service des entreprises avec des moyens donnés.

L’Union Européenne a décidé de renforcer les capacités de développement et d'innovation en Europe en déployant son $7^{\mathrm{e}}$ programme cadre de recherche et développement (PCRD) et en préparant le $8^{\mathrm{e}}$ programme «Horizon 2020 » centré sur l'innovation et la connaissance pour la croissance. Des plates-formes technologiques européennes ont été créées pour faciliter l'identification des verrous technologiques et des problématiques techniques rencontrés par les acteurs économiques, et en particulier les PME, et faire en sorte que les actions de recherche financées répondent au mieux aux attentes des entreprises et des marchés. C'est le cas de la plate-forme MANUFUTURE, plus particulièrement destinée à ce que soient conservées en Europe des capacités de production et des compétences sur les systèmes de production. Une implication forte des acteurs professionnels mécaniciens dans ces réseaux européens de recherche permet la constitution de relais et de compléments substantiels à travers le montage de projets de R\&D européens facilitant la prise en compte des attentes françaises. C'est aujourd'hui une mission importante de la plate-forme MECAFUTURE. 
Consciente de l'importance de nouer et développer les liens les plus forts avec la communauté scientifique, l'Organisation professionnelle mécanicienne a toujours eu à cœur de développer un réseau structuré avec les représentants du monde académique. Le Haut Comité Mécanique a été créé suite à une proposition d'un rapport de l'Académie des sciences sur la mécanique publié en 1981. Abrité par la FIM, il est composé d'industriels et de partenaires d'organismes de recherche et d'universitaires, et constitue une autorité morale très enracinée dans les différents tissus représentatifs de la mécanique française. Le HCM situe ses réflexions et ses initiatives, de nature stratégique, dans le cadre d'une vision globale de ce que doit être la mécanique dans une société contemporaine «au cœur du monde en mouvement " : une discipline scientifique présente dans de nombreux domaines, des plus fondamentaux aux plus proches des besoins immédiats du «terrain », et dans les champs d'application les plus vastes, avec une approche plus technique qui, par le biais de la conception et de la réalisation d'objets et de systèmes de plus en plus complexes, développe une aptitude permanente à l'intégration des savoirs et des fonctions au service des entreprises et des besoins du pays.

La FIM a, par ailleurs, activement contribué à la création en 1997, aux côtés du HCM, du CETIM et de l'AUM (Association universitaire de mécanique), de l'Association Française de Mécanique (AFM). Cette société savante réunit des ingénieurs de l'industrie, des chercheurs et des enseignants qui partagent leurs connaissances et leurs compétences pour développer la recherche et les technologies au profit des industries. L'AFM constitue une plate-forme d'échanges entre les membres des communautés industrielles et universitaires, visant à renforcer la position de la mécanique dans les systèmes de formation, de soutien et d'évaluation de la recherche, menant des actions de promotion de la mécanique auprès des pouvoirs publics français et européens, et coopérant avec des sociétés savantes françaises et internationales.

\subsubsection{Des nouveaux métiers, une image à améliorer}

L'exigence d'innovation industrielle requiert des niveaux de qualification de plus en plus élevés, permettant ainsi la création d'un cercle vertueux de développement des compétences et d'accélération de la performance. On voit ainsi émerger des métiers répondant aux nouvelles demandes des entreprises de la mécanique, tels les concepteurs de solutions globales, les coordonnateurs d'équipes éclatées géographiquement, juridiquement et culturellement (acheteurs, coordonnateurs, chefs de projet, chefs de chantier, etc.), les mécatroniciens, les opérateurs d'éolien, les électromécaniciens ou les ingénieurs en écoconception. En créant de nouveaux besoins, la mécanique développe de nouvelles compétences et stimule l'emploi. Les mécaniciens doivent prendre les devants et créer les formations qui feront émerger les compétences recherchées sur le terrain, comme les licences professionnelles...

La culture industrielle française est le fruit d'une histoire, d'un enracinement. C'est une culture de la performance, du développement humain et du progrès technique. Négligée par les jeunes, l'industrie française, et notamment sa filière mécanique, prend conscience de l'urgence d'inverser ce mouvement. Pour les 
attirer, il faut avant tout pallier le déficit d'image de la mécanique, faire connaître ses métiers, sa créativité et ses parcours professionnels. Des ambassadeurs de tous les secteurs de la mécanique doivent aller à la rencontre des jeunes pour raconter leurs expériences. La France a toutes les cartes en main : des techniciens ultraqualifiés, des élites de haut niveau, des infrastructures modernes. Toutefois, il faut réaffirmer la fierté de la communauté mécanicienne, valoriser ses expertises, ses Hommes et ses succès.

\subsubsection{Le mécanicien $d u X X I^{e}$ siècle au service de la société : les défis à venir... l'usine du futur}

La société doit se préparer à relever trois grands défis au cours des prochaines décennies :

- le défi de la démographie et de la consommation : 9 milliards d'êtres humains vivront sur la planète en 2050, constituant des enjeux majeurs en termes :

- d'alimentation (productivité agricole, hygiène et qualité des process et produits alimentaires...),

- de gestion de l'eau (problématiques de raréfaction (économie), de désalinisation, d'accessibilité, d'épuration, de stockage, de recyclage...),

- de santé (vieillissement de la population et problématique de dépendance, d'amélioration du confort de vie, nouveaux outils de diagnostics et d'intervention...),

- de logement (économies d'énergie, d'eau (pompes/robinets, tuyauterie...), diminution des pollutions, déconstruire plutôt que démolir...) ;

- le défi du développement durable, constituant des enjeux majeurs en termes :

- d'écoconception (moins de matière, moins d'énergie, moins de déchets...),

- d'écoproduction (réduction des pollutions - captage et stockage du $\mathrm{CO}_{2}$, recyclage des déchets...),

- d'énergie (limitation de la consommation, accroissement de la production par sources alternatives et renouvelables, réduction des gaz à effet de serre...),

- d'énergies renouvelables (éolien, hydraulien, biomasse...) ;

- le défi de la mobilité, de la communication et de la connectivité, constituant des enjeux majeurs en termes de :

- transport : augmentation des besoins de mobilité tout en garantissant sécurité et rapidité et en diminuant les effets négatifs sur l'environnement (automobile hybride/électrique, TGV, aéronautique plus performante, naval grande capacité...),

- nouvelle organisation des flux industriels : navires usine déportant en mer les procédés industriels indésirables sur terre (centrales énergétiques, traitement des déchets, chimie, pétrochimie...),

- circulation accélérée de l'information et des personnes, connectivité... 
L'innovation doit être, plus que jamais, comprise comme un élément clé de l'ADN de la mécanique française. C'est grâce à son inventivité et à sa créativité que la mécanique française saura trouver les solutions pour répondre à ces grands défis industriels de demain. Il n'est donc de développement possible des secteurs de haute technologie ou de la recherche fondamentale que sur la base d'un socle mécanicien solide. Demain, ce seront les nanotechnologies, les biotechnologies, les nouveaux matériaux, les équipements pour réduire l'impact environnemental ou produire de l'énergie autrement, les robots pour l'aide à la personne, qui auront plus encore besoin des compétences mécaniciennes. Avec la révolution environnementale et énergétique qui se prépare, le monde de demain sera plus que jamais demandeur de solutions industrielles largement issues de la mécanique : qu'il s'agisse de produits et procédés consommant moins de ressources naturelles devenues rares ou de substitution de technologies ou de matériaux satisfaisant aux impératifs du développement durable. Au-delà de l'intégration " mécatronique » aujourd'hui largement pratiquée, l'industrie mécanique, qui apparaît comme mature, devra organiser une interaction forte de ses technologies avec celles de la chimie et de la biologie.

Pour répondre à l'obligation grandissante de la protection de l'environnement, l'industrie se donne, pour les années qui viennent, une ambition forte fondée sur deux constats. Sa propre compétitivité et donc sa durabilité dépendront de plus en plus de son souci d'économie d'énergie et de matières premières mais aussi, sur le plan international, de son image de " partenaire » de l'écologie. Les progrès dans la protection de l'environnement ne pourront s'effectuer sans le recours à de nouveaux procédés, techniques et équipements industriels, et donc sans croissance des efforts de recherche et d'innovation dans ce domaine.

D’une manière générale, ces orientations stratégiques se retrouvent dans le concept d'usine du futur. Les industriels mécaniciens étant les principaux fournisseurs des équipements de production de toutes les filières industrielles, l'organisation professionnelle mécanicienne, associée à ses partenaires technologiques CETIM, IS et AFM, a mis en place un grand projet fédérateur destiné à affirmer son rôle de référent en matière d'usine du futur. Ce grand projet aura pour vocation d'aider les chefs d'entreprise à s'approprier le concept et construire des usines du futur, à accompagner les pouvoirs publics dans leurs différents programmes de soutien aux entreprises dans le cadre de l'usine du futur et à contribuer, auprès des acteurs de la recherche, à ce que les nombreux programmes de recherche sur le sujet répondent aux besoins des industriels et de l'économie du pays.

\subsection{Les technologies prioritaires}

Dans le cadre de sa mission d'anticipation technologique, le CETIM réalise depuis 1997 un exercice de prospective « technologies prioritaires en mécanique », qui a pour objectif d'identifier les technologies pouvant apporter un avantage compétitif aux entreprises de la mécanique.

Mené tout d'abord en écho à l'exercice «technologies clés » du ministère de l'Industrie, «technologies prioritaires en mécanique » est devenu, au fil des 
années, une démarche dynamique et reconnue permettant l'identification de pistes de réflexion stratégique, individuelle et collective, de l'ensemble des entreprises mécaniciennes françaises et des acteurs économiques en charge du développement de cette filière transversale.

Grâce à un groupe de pilotage actif, réunissant la FIM, ainsi que d'importants industriels de la mécanique, des représentants du monde universitaire et d'autres centres techniques (LRCCP, Institut de soudure, CETIAT), le CETIM est en mesure non seulement de publier tous les cinq ans un ouvrage, mais surtout de mettre à jour chaque année (sous format électronique) une version «up to date» de ces technologies prioritaires en mécanique.

Cette mise à jour annuelle en fait l'un des très rares exercices de prospective technologique au monde à être aussi dynamique. Elle permet de s'adapter à la fois au rythme (de plus en plus rapide) d'évolution des technologies, et à celui des innovations que doivent proposer les entreprises de la mécanique à leurs clients.

Toujours dans le souci de rendre cet exercice de prospective facile à exploiter, le nombre de technologies prioritaires est volontairement limité à une quarantaine. Et la priorité est donnée à des technologies diffusantes, c'est-à-dire accessibles, à court ou moyen terme, à des PME.

\subsubsection{Méthodologie}

Les technologies prioritaires en mécanique sont identifiées en analysant :

- les évolutions et progrès scientifiques et technologiques au niveau international, vus à travers les travaux de recherche et les roadmaps,

- les exigences et besoins des grands secteurs clients de la mécanique (automobile, aéronautique, énergie...),

- l'évolution du contexte réglementaire et politique français,

- les apports des fournisseurs des secteurs mécanique et informatique (matériaux, capteurs, logiciels...).

Le CETIM et ses partenaires exploitent ainsi leur vision multifilière et multitechnologie pour identifier des technologies dans divers domaines, qui tendent à déborder de plus en plus du périmètre mécanicien, vers l'électronique ou les biotechnologies par exemple.

Les technologies retenues comme prioritaires doivent avoir un caractère transversal (impact de plusieurs professions de la mécanique), ne sont pas forcément développées par des mécaniciens et peuvent constituer une innovation de rupture, mais aussi (et le plus souvent) une innovation incrémentale.

Chaque technologie prioritaire est présentée dans une fiche de deux pages, donnant l'essentiel de ce qu'il faut connaître pour appréhender la technologie.

Ces technologies prioritaires sont classées en sept briques, correspondant aux grands domaines d'innovation d'une entreprise de mécanique :

- entreprise performante,

- développement durable, 
- matériaux et surfaces,

- conception et simulation de produit et procédé,

- procédés de fabrication,

- mécatronique,

- contrôle et surveillance.

Ces technologies prioritaires en mécanique répondent à des objectifs majeurs : compétitivité, réduction de la consommation d'énergie, fiabilité, allègement...

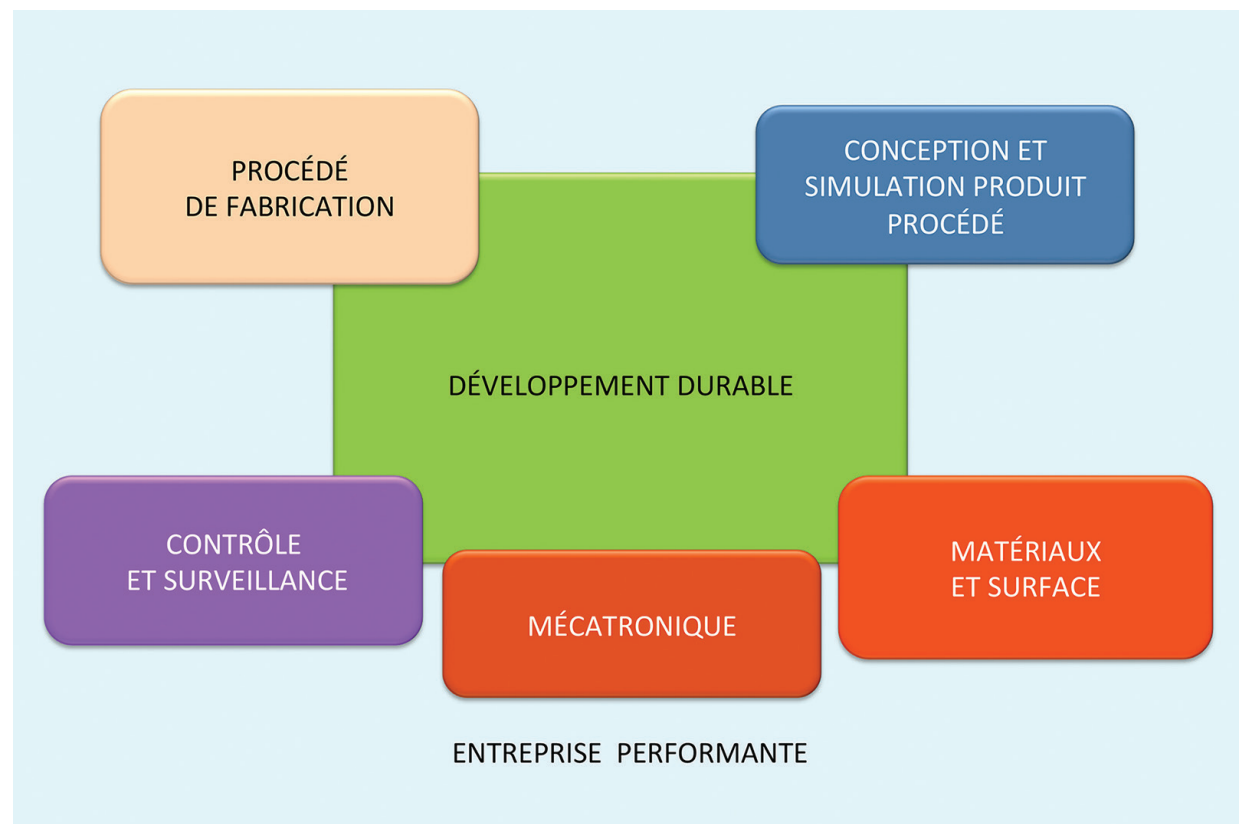

Figure 1.5. - Les sept briques des technologies prioritaires en mécanique.

\subsection{2. « Entreprise performante 》}

Il s'agit des moyens informatiques (méthodes et outils) qui contribuent à la performance de l'entreprise en termes de productivité, de compétitivité et de visibilité sur le marché. Les évolutions concernent l'informatique décentralisée distribuée, les méthodes et outils de la performance, les services et applications techniques mobiles, les offres de services en mode cloud et la gestion de l'information stratégique : veille et visibilité.

On note également les plans stratégiques lancés aux États-Unis (plan Obama), en Allemagne et en France pour dynamiser les industries manufacturières dans ces pays. En France la vision « usine du futur» et en Allemagne le plan stratégique Industrie 4.0 visent à mettre en place des machines intelligentes, les procédés robotisés et appliquer l'intelligence collaborative aux moyens de production. 


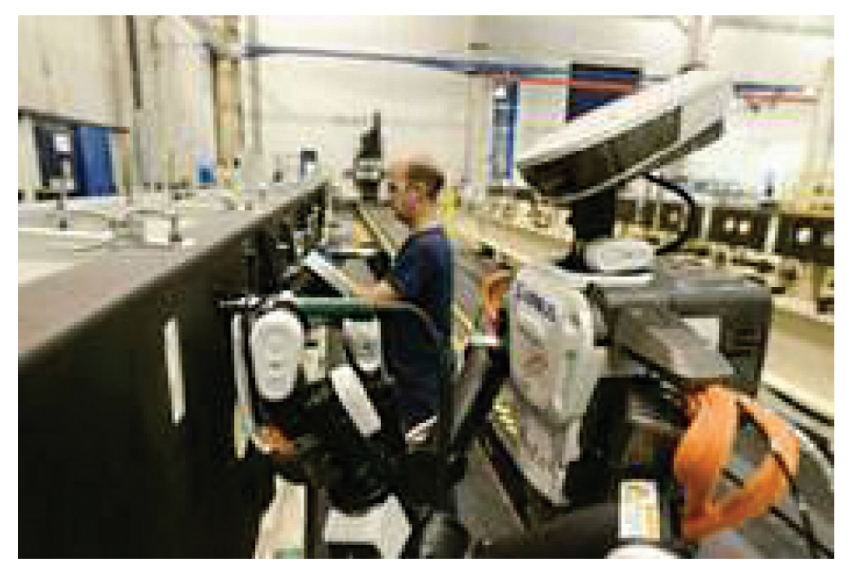

Figure 1.6. - Un robot collaboratif sur les chaînes d'assemblage Airbus.

La nouvelle technologie prioritaire "applications techniques mobiles ", introduite en 2014, traduit le développement très rapide des outils et services disponibles sur tablettes et smartphones, tant pour le calcul ou la simulation en bureau d'études que pour le pilotage de machines.

On note également un fort intérêt des entreprises de la mécanique pour améliorer leur démarche interne de veille.

\subsection{3. «Développement durable 》}

Portée par la prise en compte des problématiques de l'environnement et de la maîtrise de la consommation énergétique ainsi que des besoins sociétaux, les technologies qui ont le plus évolué concernent :

- l'écoconception, avec l'apparition des premières directives (EuP) et des premières normes spécifiques aux produits de la mécanique, ainsi qu'une sensibilisation accrue des donneurs d'ordres vis-à-vis de la performance environnementale des produits achetés ;

- la réduction de la consommation énergétique de produits et de procédés est également portée par un contexte réglementaire fort avec les objectifs issus du Grenelle de l'environnement pour réduire les émissions de $\mathrm{CO}_{2}$;

- le développement des énergies renouvelables visant à améliorer la performance technique et économique des énergies éoliennes, marines et photovoltaïques.

Enfin, l'ajout de deux technologies sur les bioproduits, qui intègrent des matériaux ou substances issus du végétal, et les bioprocédés, où des procédés biochimiques se substituent à des procédés mécaniques.

L'efficacité énergétique est un axe fort de préoccupation dans l'industrie mécanique, tant au niveau des produits que des procédés. Les biomatériaux progressent également. 


\subsection{4. « Matériaux et surface »}

Les évolutions marquantes des technologies de la brique "matériaux et surface " concernent en premier lieu les composites thermoplastiques, les procédés de fabrication à grande cadence des pièces en matériaux composites pour les applications de transports terrestres et la mécanique, le traitement de surface des fibres pour une meilleure adhésion fibre/matrice pendant la mise en œuvre et le développement des résines thermoplastiques fluides. Ces évolutions sont fortement liées aux procédés de mise en œuvre des composites à fibre longue notamment.

En ce qui concerne les matériaux composites, les évolutions sont orientées vers le développement des matériaux composites biosourcés et leur mise en œuvre, nouveaux polymères pour le procédé RTM ou pultrusion, les composites à matrice céramique.

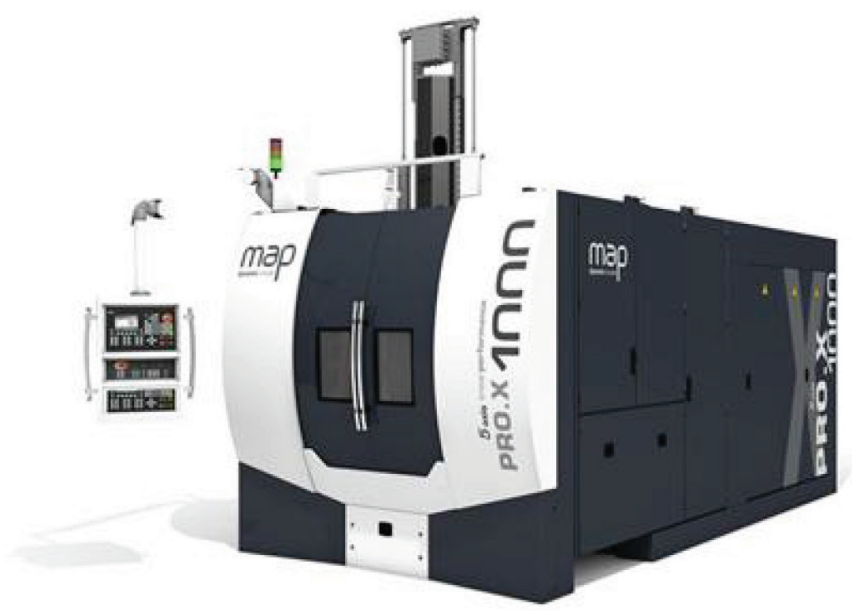

Figure 1.7. - Les composites dans les structures des machines d'usinage.

Les autres évolutions concernent :

- la simulation des procédés (formage, RTM) ;

- le couplage des procédés, notamment le formage-injection pour fabriquer les pièces hybrides multimatériaux ;

- la dépollution et le nettoyage par procédés physiques (plasma froid, savon magnétique) ;

- les matériaux résistants à la corrosion sans traitement.

À côté des matériaux métalliques qui poursuivent leurs progrès et des matériaux composites dont les applications structurales se développent, on note l'émergence de multimatériaux. 


\subsection{5. "Conception et simulation de produit et procédé "}

Les développements les plus marquants ont été réalisés autour de l'introduction des approches fiabilistes en conception et leur mise en ouvre pour les applications industrielles.

L'introduction du prototype virtuel et de la réalité virtuelle augmentée concrétise la généralisation des approches numériques en conception et en procédés de mise en œuvre.

Plusieurs technologies ont été introduites au cours de ces dernières années :

- le vieillissement et la durabilité des polymères et composites correspondent à la prise en compte des problématiques d'endommagement spécifiques à ces matériaux, en liaison avec la progression de leur utilisation en mécanique ;

- la conception pour l'assemblage, la séparabilité et le montage intègre à la fois une réflexion sur la conception modulaire et la prise en compte de la chaîne logistique et de la fin de vie des produits ;

- la simulation multiéchelle qui est de plus en plus couplée à l'approche multiphysique, pour affiner les simulations de phénomènes complexes ;

- la simulation du procédé et le lien avec la conception des produits pour optimiser la phase d'industrialisation et améliorer la qualité des produits.

Les évolutions plus récentes pour la brique « conception et simulation » sont en lien avec l'innovation collaborative ou open innovation, l'éco-innovation comme une opportunité de différenciation et/ou comme un outil pour la prise en compte des réglementations environnementales, le dimensionnement par rapid sizing et le développement des méthodes de la réduction de modèles permettant de réaliser des calculs paramétriques à moindre coût.

La nouvelle technologie prioritaire «conception biomimétique (identifier des solutions en s'inspirant du vivant) commence à trouver des applications industrielles intéressantes et variées.

Dans une approche plus classique de la conception, et avec le développement de la simulation multiphysique, la notion de chaînage numérique devient prioritaire.

\subsection{6. «Procédés de fabrication »}

Les technologies de la brique " procédés de fabrication » ont sensiblement évolué ces dernières années.

Pour la technologie assemblage multimatériaux, les évolutions concernent les techniques de collage, notamment pour la caractérisation d'assemblages métal/ composite. Les procédés de mise en ouvre des composites sont traités dans la brique «matériaux et surface ».

La fabrication additive de pièces métalliques s'est développée grâce à la mise sur le marché de machines et de matériaux permettant de répondre aux besoins de l'industrie sur des pièces complexes en petites séries. Les évolutions majeures pour cette technologie concernent la disponibilité de machines d'impression 3D à bas coût, la machine combinée frittage laser/fraisage à grande vitesse et la 
technique micro MIM (Metal powder Injection Molding) pour fabriquer des pièces complexes en métal.

Le manufacturing fait l'objet d'un intérêt fort des industriels et des pouvoirs publics au niveau européen, notamment les travaux de l'association EFFRA (European Factories of the Future Research Association) en lien avec les besoins de la recherche et développements exprimés dans la feuille de route intitulée "Factories of the Future ». Ces travaux ont permis d'initier la vision « usine du futur» en France comme le plan allemand «industrie 4.0 ».

La fabrication additive est assurément la plus grande innovation de rupture que connaît la mécanique actuellement, avec un développement exponentiel des possibilités. Les applications de la robotique et les machines intelligentes se développent parallèlement.

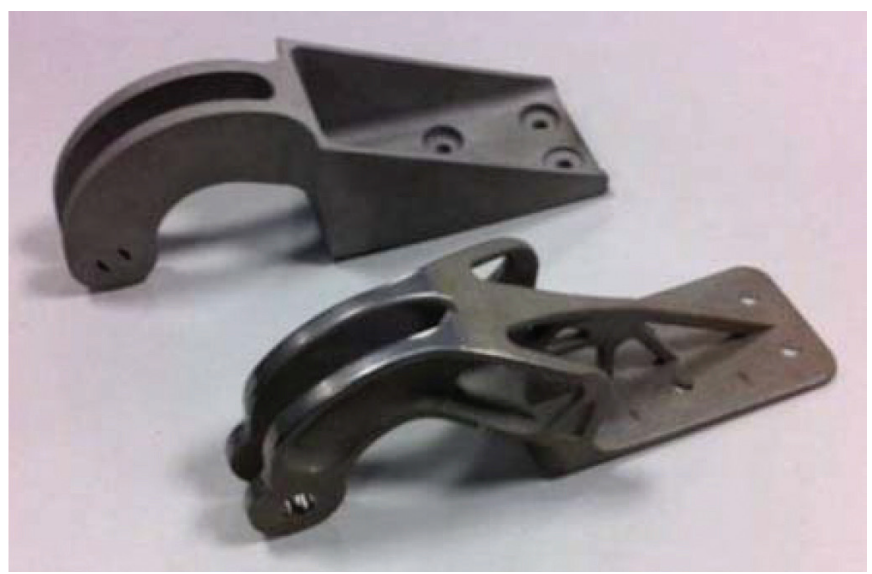

FigURE 1.8. - Fabrication additive, véritable opportunité en aéronautique.

\subsection{7. «Mécatronique »}

Il s'agit de la brique qui porte les technologies qui ont le plus fortement impacté la conception des produits mécaniciens.

Les deux technologies qui ont le plus progressé concernent en premier lieu la gestion de l'énergie embarquée qui assure l'autonomie des systèmes et une meilleure maîtrise de la consommation énergétique. En second lieu, l'émergence de l'adaptronique est constatée, avec le passage des travaux de R\&D en amont vers les produits industriels, pour intégrer des actionneurs directement dans les composants et les structures.

L’intégration des capteurs se généralise dans les composants et équipements, y compris pour des applications en environnement sévère avec le développement de capteurs adaptés.

L'utilisation des actionneurs électriques progresse grâce à leur augmentation de puissance et l'utilisation de lois de commandes avancées plus efficaces, notamment pour les applications aéronautiques. 
Les technologies de la communication sans fil sont devenues plus matures, gagnant en fiabilité et en vitesse de transmission de l'information.

Pour la technologie adaptronique, les évolutions récentes ont concerné les suspensions actives pour améliorer le confort en réduisant les vibrations, la récupération d'énergie par actionneur inverse et la récupération et l'utilisation de la chaleur des moteurs.

Les notions d'objets communicants et d'adaptronique deviennent omniprésentes, y compris en mécanique. Et le développement de la mécatronique pose la question de la tolérance aux pannes de ces systèmes.

\subsection{8. "Contrôle et surveillance des systèmes de production »}

Les évolutions pour la technologie capteurs, surveillance, CND ont concerné d'une part les méthodes intelligentes de traitement des données, les méthodes de CND par combinaison des techniques comme l'électromagnétisme et les ultrasons et la mise sur le marché de capteurs bas coût pour les applications de détection de la contamination des fluides hydrauliques ou la détection de présence. Les techniques CND pour la détection de défauts dans les matériaux composites et des assemblages multimatériaux se développent avec un intérêt fort de la part des industriels, notamment l'aéronautique.

D’autres évolutions plus récentes en lien avec les capteurs et le traitement des données concernent la miniaturisation des capteurs, les composants avec fonction de surveillance intégrée et les applications de surveillance sur tablette.

On peut citer également la corrélation d'images numériques parmi les nouveaux axes dans cette brique.

Les technologies prioritaires en mécanique sont mises à jour régulièrement et sont disponibles sur le site Internet du CETIM (www.cetim.fr).

\section{Annexe : liste des technologies prioritaires en mécanique}

\section{ENTREPRISE PERFORMANTE}

Informatique décentralisée distribuée

Méthodes et outils de la performance

Veille stratégique

Entreprise étendue

Intégration du facteur humain

\section{DÉVELOPPEMENT DURABLE}

Biomatériaux

Écoconception

Usines du développement durable, écofabrication

Formage et usinage écologiques

Procédés propres de préparation et traitement de surfaces

Efficacité énergétique des produits et procédés 


\section{MATÉRIAUX ET SURFACES}

Aciers très hautes performances et superalliages

Alliages légers et de titane

Composites thermoplastiques

Élastomères nanochargés

Surfaces fonctionnelles

\section{PROGÉDÉS DE FABRICATION}

Intégration des robots en production

Procédés de formage near net shape

Usinage hautes performances

Fabrication directe de pièces métalliques ou polymères

Microfabrication

Systèmes de production agiles et adaptatifs

Assemblages multimatériaux

Techniques de soudage à hautes performances

Technologies innovantes de formage des tôles

\section{CONGEPTION ET SIMULATION DE PRODUIT ET PROGÉDÉ}

Conception et fabrication intégrées

Conception de produits fiables et sûrs

Méthodes innovantes de conception

Simulation et optimisation numériques produit ou procédé

Spécification géométrique des produits

Conception biomimétique

\section{MÉCATRONIQUE}

Conception/simulation mécatronique

Capteurs autonomes et communicants

Commande et contrôle avancés

Composants et machines communicants

Adaptronique (smart materials)

\section{CONTRÔLE ET SURVEILLANGE}

Surveillance en continu

Techniques avancées de CND

Télésurveillance et pilotage à distance

Traçabilité

\subsection{Un atout majeur pour l'innovation et l'emploi des jeunes}

La mécanique est le premier employeur industriel de France avec 628000 salariés.

Le savoir-faire et la compétence du personnel est au cour de la compétitivité de la mécanique.

Les métiers de l'industrie doivent lutter contre leur mauvaise image pour attirer des compétences et être en mesure de faire face aux besoins de recrutement 
auxquels les entreprises sont confrontées. Elles doivent s'ouvrir aux jeunes, aux familles, aux écoles...

Le développement de l'apprentissage et de l'alternance et le renforcement des liens avec l'Éducation nationale, les universités, etc., sont nécessaires pour favoriser l'intérêt des jeunes pour nos filières et ne pas bloquer les entreprises dans leur croissance faute de personnel qualifié.

Dans cette perspective, le comité FER (Formation, Emploi, Recrutement) de la FIM a pour rôle et pour missions :

- améliorer la compétitivité des entreprises de la mécanique en leur permettant de disposer du personnel qualifié dont elles ont besoin ;

- coordonner les initiatives prises par les diverses professions de la mécanique en matière de formation et de valorisation des métiers ;

- assurer le lien avec l'appareil éducatif public ou professionnel en matière de formation.

\subsubsection{La mécanique et l'emploi : tendance à venir et besoins}

Les industries de la métallurgie, dont les industries mécaniques représentent une très large part, recrutent en moyenne entre 60 et 80000 personnes par an, dont 30 à $40 \%$ de jeunes ; 40 à 50000 le sont dans les industries mécaniques.

Ces opportunités sont le résultat d'une vague massive de départs à la retraite et de la nécessité pour les entreprises de relever les défis mondiaux de l'innovation pour rester compétitives.

Le mécanicien est amené à innover à la demande de ses clients en s'intéressant de plus en plus près à leurs métiers et à leurs besoins. Cela l'amène à fournir, non plus un produit ou un service isolé, mais une fonction complète dont il garantit la performance. Cela induit une augmentation notable d'emplois de plus en plus qualifiés.

La hausse du niveau de qualifications est une tendance lourde. Si historiquement, l'industrie recrutait majoritairement des personnels de niveau CAP ou BEP, la tendance s'est inversée ces dernières années au profit des niveaux de formation supérieurs, y compris pour les postes d'employés ou d'ouvriers qualifiés. Les baccalauréats et les baccalauréats professionnels devraient représenter à terme $25 \%$ des recrutements, les BTS et les DUT $23 \%$ et les masters et autres doctorats $17 \%$.

En dix ans, la proportion des ouvriers hautement qualifiés est passée de 15 à $22 \%$. Dans le même temps, le nombre d'ingénieurs a grimpé de $50 \%$. Ils représentent aujourd'hui $21 \%$ des salariés des entreprises (source : UIMM).

\subsubsection{Les types de profils recherchés}

Conséquence de la montée des niveaux de compétences et de l'adaptation des formations initiales au développement des innovations: l'émergence de nouveaux métiers tels que les concepteurs de solutions globales («architectes", 
intégrateurs) et les coordonnateurs d'équipes éclatées géographiquement, juridiquement et culturellement (acheteurs, coordonnateurs, chefs de projet, chefs de chantiers, etc.).

Les équipes monodisciplinaires disparaissent progressivement au profit d'équipes montées par projet.

Par ailleurs, les fonctions relatives à la réalisation d'outillages, au redéploiement des machines, à la programmation des commandes numériques ou encore à la maintenance préventive ou curative des machines prennent toujours plus d'importance pour améliorer l'adaptabilité rapide des outils de production à une nouvelle commande et pour éviter tout blocage coûteux de la fabrication.

Quelques métiers émergents :

- les métiers de la recherche et du développement, notamment dans l'automobile, le naval, l'aéronautique et le ferroviaire, et les équipements mécaniques ;

- les métiers de l'électronique et de la mécatronique ;

- les métiers du contrôle et de la qualité ;

- la robotique ;

- la méthode, les process de fabrication ;

- les métiers liés aux composites ;

- la maintenance ;

- les métiers liés à l'environnement.

\subsubsection{Les formations et l'apprentissage en mécanique}

L'éventail des formations est large ; il s'étend du CAP en alternance au bac +5 ingénieur et plus. Cinquante-et-un centres de formation des apprentis de l'industrie (CFAI), répartis sur l'ensemble du territoire, forment chaque année par la voie de l'apprentissage industriel plus de 25000 apprentis aux bacs professionnels, BTS et licences pro. Plus de 1700 ingénieurs sont diplômés tous les ans des 23 ITII (instituts des techniques d'ingénieurs de l'industrie).

Il existe aujourd'hui trois filières pour accéder aux emplois de la mécanique : les formations à temps plein de l'Éducation nationale, les contrats de professionnalisation et les contrats d'apprentissage. Chaque année, plus de 36000 jeunes de 16 à 26 ans, garçons et filles, signent ces types de contrats. Les premiers permettent d'obtenir en six à douze mois un Certificat de Qualification Professionnelle de la Métallurgie (CQPM). Les seconds préparent sur un, deux ou trois ans un diplôme de l'Éducation nationale ou un titre d'ingénieur.

Les entreprises de mécanique accordent une très grande place à la formation et à l'apprentissage : à la fois comme voie d'accès privilégiée à l'emploi dans ces entreprises pour les jeunes et comme moyen d'améliorer les compétences et les savoir-faire des salariés en poste. On parle d'entreprises «apprenantes ». Via les dispositifs de formation continue, de responsabilité des équipes, ces métiers favorisent l'ascenseur social : ces entreprises permettent de passer d'un BEP à un bac pro, puis BTS, puis ingénieur.

L'effort de formation est supérieur à la moyenne nationale. 
L'apprentissage est également une excellente porte d'entrée dans l'entreprise, et débouche très souvent sur une embauche. Selon une enquête 2010 de l'UIMM effectuée sur les sortants de juin-septembre 2009, 50 \% des apprentis sont restés dans leur entreprise d'accueil avec un nouveau contrat de travail. Parmi l'autre moitié, $43 \%$ ont mis moins d'un mois pour trouver un emploi dans une autre entreprise et $35 \%$ entre un et trois mois.

\subsubsection{L'attractivité des métiers}

- Enjeu : le paradoxe entre le déficit d'image des industries mécaniques et le dynamisme et l'attractivité de leurs métiers.

- Les atouts des métiers de la mécanique : des emplois stables, des rémunérations attrayantes, des perspectives d'évolution de carrière.

- La FIM et ses professions s'investissent pour promouvoir les métiers de la mécanique : organisation de colloques, participation à des salons, réalisation d'outils pédagogiques pour présenter les métiers, actions spécifiques aux professions...

Si la crise a touché durement certaines entreprises de mécanique, d'autres continuent actuellement à rechercher des collaborateurs et doivent préparer les recrutements à venir.

L'industrie souffre aujourd'hui d'un grave déficit d'image qu'il faut corriger car il ne permet pas de percevoir la réalité des entreprises mécaniciennes.

En effet, l'image vieillissante des industries mécaniques, en contradiction avec son dynamisme et son modernisme, doit être cassée pour pallier les conséquences en termes d'attractivité, de relève de la main d'œuvre et de transmission des savoir-faire.

Un facteur pénalisant, et peut-être le plus crucial, est la motivation ou plutôt son absence. Les jeunes ne sont pas attirés par les métiers de l'industrie et peu orientés vers les filières professionnelles considérées comme dévalorisantes, notamment par ceux qui ont connu le monde de l'usine à une époque où les conditions physiques de travail étaient difficiles.

Par conséquent, le vivier de jeunes pour les métiers industriels, et plus particulièrement de la mécanique, se tarit d'année en année.

L'enjeu est capital car les difficultés subsistantes en période de crise vont s'amplifier lors de la reprise et risquent de bloquer les entreprises dans leur croissance.

Les problèmes de formation ne peuvent être résolus par une politique de court terme. La persévérance est de rigueur. Poursuivre les campagnes de mobilisation des jeunes, introduire et valoriser de nouvelles formations en mécanique dans les universités et collèges avec une pratique plus généralisée de l'alternance, accroître les moyens de formation technique permanente pour permettre une meilleure adaptation des «seniors ", mieux coordonner les écoles d'ingénieurs en mécanique sont autant de leviers pour répondre à l'enjeu des compétences indispensables aux entreprises mécaniciennes. 


\subsubsection{Les atouts des métiers de la mécanique}

Les emplois offerts par les entreprises de mécanique sont relativement stables : « $92 \%$ des salariés de la métallurgie sont en contrat à durée indéterminée, contre $84 \%$ pour l'ensemble des secteurs et $83,4 \%$ dans le tertiaire. »

En plus d'offrir des emplois stables, le niveau de rémunération des métiers industriels n'est pas négligeable, comparé notamment à certains salaires dans les services : sous l'effet de la hausse des qualifications et de la baisse proportionnelle de l'emploi non qualifié, les entreprises de mécanique semblent offrir des emplois bien rémunérés (de 1500 à 2200 euros pour les opérateurs/ouvriers qualifiés ; de 1700 à 3100 euros pour un technicien ou un agent de maîtrise; de 2600 à 3400 euros pour un ingénieur ou cadre débutant - source : UIMM - ; puis, de 3000 à 5000 euros pour un ingénieur ou cadre en fonction de la mission et du niveau de responsabilité ou d'expertise).

Enfin, les évolutions de carrière sont très importantes dans les emplois qu'offrent nos entreprises, ce qui est un gage de mise à niveau constante des compétences. Dans la métallurgie, 430000 salariés sont concernés chaque année (18 millions d'heures) par la formation continue (source : UIMM). Le développement de compétences professionnelles nouvelles permet à chacun de progresser dans son métier, favorise la mobilité interne et accompagne les réorientations.

La FIM et les professions qu'elle regroupe agissent chaque jour pour valoriser l'image de leurs métiers afin d'inverser la tendance. L'enjeu est de taille : permettre aux entreprises mécaniciennes de disposer des compétences dont elles ont besoin pour être compétitives.

Quelques exemples d'actions menées par la FIM et ses professions :

- l'organisation en octobre 2011 d'un colloque «Choisir la mécanique : des métiers d'avenir» pour faire découvrir au monde éducatif la richesse des industries mécaniques et de ses métiers, et réfléchir ensemble aux moyens à mettre en place pour développer les liens entre l'Éducation nationale et les entreprises de ce secteur ;

- Jérôme Frantz, président de la FIM, et Pascal Charvet, directeur de l'ONISEP, ont signé en mars 2013 à la Maison de la mécanique une convention de partenariat pour une durée de trois ans. Objectif : contribuer à valoriser les métiers de la mécanique auprès du grand public par le biais de ses syndicats membres ;

- plusieurs professions de la mécanique et un centre de formation participent chaque année sous l'égide de la FIM au salon l'Aventure des métiers pour promouvoir auprès des jeunes les métiers de la mécanique. Sur cet espace dont le slogan est «Industries mécaniques : faites bouger le monde! », les visiteurs peuvent découvrir concrètement les métiers de la mécanique au travers de diverses animations : un simulateur de soudage, une maquette de matériel de manutention simulant des pannes, un atelier de production taille réduite... ;

- en 2012, plus de 1000 jeunes, collégiens et lycéens ont participé à l'une des interventions organisées par la FIM et le CETIM pendant la Semaine de l'industrie dans 14 établissements scolaires de France ; neuf régions au total étaient concernées. 
À cette occasion, un quiz ludique et interactif «La mécanique au cour de la vie » a été lancé. Cet outil pédagogique a pour objectif de permettre aux industriels, enseignants... de présenter les métiers de la mécanique dans les établissements scolaires au travers de cinq thématiques : emploi, secteurs, métiers, produits et environnement. Plus de trente questions et de nombreuses interviews et vidéos de portraits de métiers pour comprendre leur richesse et les technologies proposées par cette industrie!

En 2013, 2760 opérations - dont 364 pour la mécanique qui arrive en tête des secteurs organisateurs d'opérations ! - sont organisées sur l'ensemble du territoire, soit $21 \%$ de plus qu'en 2012. La majorité de ces opérations sont des visites de sites industriels et des journées portes ouvertes, permettant ainsi au grand public, notamment aux jeunes et aux demandeurs d'emploi, de découvrir l'industrie du $\mathrm{XXI}^{\mathrm{e}}$ siècle, ses métiers et ses carrières.

- La FIM et le CETIM se sont associés pour réaliser un document PowerPoint animé qui présente la mécanique, ses défis et ses métiers au travers de nombreux exemples d'innovations mécaniciennes et de témoignages.

Objectif : donner un support de communication complet aux industriels qui vont présenter la mécanique dans les écoles, les collèges, les universités.

- La FIM a lancé en mars 2013 un site Internet dédié à ceux qui souhaitent découvrir la mécanique et/ou la promouvoir auprès des jeunes.

Le site www.lesmetiersdelamecanique.net offre deux entrées aux internautes :

- «Découvrir » pour trouver des informations sur la mécanique et ses métiers, ses formations, les besoins de recrutement des entreprises du secteur...;

- «Promouvoir » pour les industriels, enseignants et conseillers d'orientation psychologues qui peuvent télécharger les outils de promotion développés par la FIM : la présentation PowerPoint «Les défis de l'industrie mécanique », le quiz «La mécanique au cour de la vie », la vidéo «Au cœur de toutes les industries... La mécanique »...

Le site laisse une large place aux témoignages avec la rubrique « Portraits ». Jeunes, apprentis, chefs d'entreprise, salariés, etc., y racontent leur expérience sous forme écrite ou vidéo.

\subsection{La formation (enseignement supérieur)}

\subsubsection{Constat}

Il est difficile de faire une synthèse sur l'état de la formation en mécanique en France, les enjeux, les changements de méthodes, les points faibles et forts.

Néanmoins, il est possible de faire plus ou moins facilement des bilans purement comptables :

- les besoins chiffrés de l'industrie en termes d'emplois, qui sont publiés par les syndicats professionnels de l'industrie mécanique ; 
- les données fournies par les établissements d'enseignement supérieur (universités, IUT, écoles d'ingénieurs...) sur le nombre de diplômés formés, par niveaux, dans le domaine de la mécanique ;

- l'évolution du nombre d'offres d'emploi dans un secteur à partir des organismes de placement (APEC par exemple) ;

- les enquêtes, réalisées depuis quelque temps par les écoles d'ingénieurs et les IUT et plus récemment par les universités, sur le devenir de leurs diplômés.

Tous ces indicateurs sont pertinents et instructifs. Il faut cependant être conscient de leur limite et les utiliser avec rigueur. En effet :

- le nombre d'offres d'emploi ne donne aucune indication sur la mission confiée au recruté ou sur ses possibilités d'évolution de carrière quelques années plus tard ;

- les enquêtes de devenir peuvent souffrir d'un taux de réponses statistiquement non représentatif, car parfois renseignées en priorité par ceux qui sont en recherche d'emploi, plus disponibles pour répondre à ces enquêtes relativement longues ;

- les besoins chiffrés de l'industrie sont à considérer avec précaution, en particulier lorsqu'ils sont très précis sur la spécialité demandée. Lorsqu'ils sont calculés à partir d'une classe d'âges partant à la retraite, cela semble assez correct. En revanche, lorsqu'ils sont basés sur des prévisions de développement d'un nouveau secteur d'activités, ces prévisions sont délicates à établir avec précision et nécessitent des études très approfondies².

Il est surtout important que la formation prodiguée dans les universités et les écoles d'ingénieurs corresponde aux besoins de la profession à long terme. Pour cela, les écoles s'appuient sur des conseils de perfectionnement et les universités font intervenir de plus en plus d'industriels dans la formation. Cependant, l'horizon des industriels est souvent dans le court ou moyen terme. Les formateurs se doivent de rappeler qu'un diplômé doit être formé pour les 45 années à venir. Sa formation doit lui fournir les moyens de s'adapter, d'évoluer et bien sûr de se former/progresser tout au long de sa vie professionnelle. Si cela a de tout temps été l'objectif, aujourd'hui la vitesse à laquelle évolue notre environnement rend celui-là beaucoup plus difficile à atteindre.

L'enjeu actuel est donc de former des mécaniciens non seulement bien préparés à leur futur métier, mais surtout disposant des capacités et des compétences nécessaires pour s'adapter tout au long de leur vie professionnelle à l'évolution de leur métier. La solution pourrait sembler avoir déjà été trouvée, puisque les personnes actuellement en fin de carrière ont réussi le challenge ; il suffirait donc de reproduire le même modèle. Cependant, un élément nouveau complique

${ }^{2}$ La Cour des comptes a relevé ce point par exemple dans son rapport sur « La politique de développement des énergies renouvelables » de juillet 2013. 
sérieusement la situation : les étudiants qui entrent aujourd'hui dans l'enseignement supérieur sont très différents de ceux qui y rentraient il y a 40 ans :

- l'évolution rapide de notre société et des technologies a conduit à changer l'enseignement dans les cycles primaire et secondaire. Cela s'est traduit par l'introduction de nouveaux enseignements dès le plus jeune âge, comme l'informatique, l'éveil à l'art, etc., et la technologie, qui a en partie pris la place de la physique de base. Aujourd'hui, le bachelier a des connaissances dans de nouveaux domaines mais, comme le nombre d'heures d'enseignement n'a pas augmenté, il a dans certaines matières des connaissances moins approfondies ;

- les enseignements en mathématiques et en physique de la seconde à la terminale scientifique $\mathrm{S}$ (comparée à l'ex-terminale $\mathrm{C}$ ) ont été réduits de respectivement $26 \%$ et $19 \%$ depuis 1990, ce qui représente 150 heures et 75 heures de moins. Pour acquérir les fondements nécessaires, entre autres, à la mécanique, les élèves disposent de moins de temps. Les seuls qui peuvent réellement y parvenir sont les meilleurs ou les privilégiés qui sont, par exemple, dans des lycées ayant les moyens financiers et humains de proposer des heures d'accompagnement personnel transformées en heures de mathématiques. Les conséquences se traduisent par l'écart grandissant entre les meilleurs élèves et ceux en difficulté, comme l'a montré l'enquête PISA de 2009, hélas confortée par celle de 2012 (Programme International pour le Suivi des Acquis des élèves). La France est aujourd'hui dans la moyenne des pays de l'OCDE pour le niveau de performance en compréhension de l'écrit et en sciences, alors qu'elle était au-dessus de la moyenne auparavant ; - $20 \%$ de la population avait le baccalauréat en 1970, contre $45 \%$ en 1990, et $75 \%$ actuellement ${ }^{3,4}$. De plus, aujourd'hui, $75 \%$ des bacheliers s'inscrivent dans l'enseignement supérieur ${ }^{5}$. Par ailleurs, le nombre d'ingénieurs diplômés a plus que doublé depuis $1990^{6}$. L'admission dans l'enseignement supérieur est donc beaucoup moins sélective ;

- l'étudiant actuel a accès à un grand nombre d'informations. C'est un avantage mais cela peut présenter des effets pervers :

o l'information obtenue provient en général d'Internet; elle est souvent non contrôlée et donc parfois fausse,

o si toutefois l'information n'est pas immédiatement accessible via un moteur de recherche grand public, l'étudiant n'est pas habitué à rechercher l'information ailleurs (moteur de recherche spécialisé, livres, etc.) ou à la vérifier ;

- dès l'école élémentaire, il a été constaté que l'élève est moins apte à une concentration longue, ce qui rend difficile un apprentissage ardu, du fait

\footnotetext{
${ }^{3}$ http://media.education.gouv.fr/file/2008/82/4/chap8-9_33824.pdf

${ }^{4}$ http://www.education.gouv.fr/cid143/le-baccalaureat.html

5 http://cache.media.enseignementsup-recherche.gouv.fr/file/etat_du_sup_en_2013/41/6/ EESR_2013_Complet_116_web+index_250416.pdf

${ }^{6}$ Rapport de la Conférence des directeurs des écoles françaises d’ingénieurs (CDEFI), janvier 2014.
} 
en particulier de l'usage intensif qui est parfois fait d'Internet ou des jeux vidéo ${ }^{7}$.

Par ailleurs, les besoins de l'industrie ont évolué.

- Tout d'abord dans le cœur de métier, car le produit «mécanique » est aujourd'hui le fruit d'un procédé pluritechnologique et est de plus en plus complexe. Les compétences nécessaires pour le réaliser sont donc de plus en plus larges.

On demande au mécanicien en licence $(\mathrm{bac}+3)$ niveau assistant-ingénieur de ${ }^{8}$ :

- connaître le rôle et le champ d'application de la mécanique dans tous les secteurs : milieux naturels (océans, atmosphère, espace...), milieux industriels (nucléaire, pétrolier, alimentaire, médical...), transports (terrestre, aérien, maritime...), enjeux sociétaux (sécurité, fiabilité, économie d'énergie, procédés propres...) et «bien-être » (confort, environnement sonore...);

- maîtriser les outils fondamentaux de la mécanique : définition d'un système mécanique, approche locale ou globale, équilibre des forces, flux et bilans énergétiques, systèmes conservatifs ou non conservatifs, mécanismes et liaisons ;

- maîtriser les notions de champs de force, déplacement, vitesse, déformation et contrainte pour comprendre des exemples simples de mouvement de solides rigides ou déformables, d'écoulements de fluides et de rhéologie ;

- pouvoir expliquer qualitativement les phénomènes simples de son environnement ;

- estimer les ordres de grandeur, manipuler correctement les unités, intégrer une bonne vision de l'espace et de ses représentations, savoir isoler un système ;

- être capable de formuler un problème avec ses conditions limites, de l'aborder de façon simple, de construire une solution et de conduire une analyse critique des résultats ;

- savoir valider un modèle par comparaison de ses prévisions aux résultats expérimentaux et apprécier les limites de validité d'un modèle ;

- connaître les principales familles de matériaux et leurs propriétés mécaniques ;

- être en capacité de réinvestir les connaissances acquises dans un contexte professionnel.

\footnotetext{
7 http://www.academie-sciences.fr/activite/rapport/avis0113.pdf

${ }^{8}$ Référentiel de compétences dans le domaine "sciences, technologies, santé " pour la licence généraliste mention mécanique rédigé par le GTT-AUM de l'AFM, paru dans le livre La licence scientifique dans l'espace francophone. Essai de référentiel de connaissances et de compétences, sous la direction d'Évelyne Garnier-Zarli, ISBN : 978-2-343-01859-1.
} 
En plus de ces compétences théoriques, l'assistant-ingénieur doit avoir des compétences pratiques acquises en laboratoire concernant l'instrumentation en mécanique, ainsi que l'acquisition et le traitement des données. À savoir :

- utiliser en autonomie des techniques courantes dans le domaine du génie mécanique : faire un schéma cinématique, connaître les outils de représentation graphique (dessin industriel) et les techniques de fabrication, par enlèvement ou ajout de matière, la mise en forme, utiliser des outils de CAO (conception assistée par ordinateur) et de simulation d'un usinage en FAO (fabrication assistée par ordinateur) ;

- utiliser en autonomie des techniques courantes dans le domaine du génie civil pour l'étude des matériaux, les interactions sols/ouvrages, l'aménagement, les infrastructures, liées à la réglementation, la normalisation, les essais de laboratoire et, in situ, la justification des ouvrages ;

- utiliser en autonomie les techniques courantes dans le domaine du génie des procédés ;

- analyser, interpréter des données expérimentales, développer une argumentation et rédiger un rapport de synthèse ;

- être en capacité d'investir ses savoirs et savoir-faire dans la maintenance industrielle.

À l'ingénieur mécanicien $(\mathrm{bac}+5)$, on va demander non seulement de mâ̂triser un certain nombre de concepts, outils et techniques susmentionnés, mais également d'être capable de les faire évoluer. Prenons l'exemple de la conception assistée par ordinateur. Il n'est pas envisageable aujourd'hui de former quelqu'un au niveau bac +5 qui ne maîtrise pas la CAO. Or la CAO est tout d'abord de la conception et ce n'est pas son apprentissage qui est assisté par ordinateur mais son optimisation (possibilité de simulation de l'objet technique, gestion des données techniques...). L'apprentissage nécessite d'acquérir les fondements de la conception. Le temps nécessaire est donc incompressible. De plus, la cotation fonctionnelle n'est pas non plus facilitée par la CAO. Le gain de temps qu'apporte la CAO dans l'apprentissage se traduit par la suppression du temps nécessaire à la mise en plans qui était antérieurement réalisée manuellement. En conclusion, l'apprentissage lié au logiciel de CAO nécessite du temps supplémentaire.

- De plus, l'industriel demande des connaissances/compétences autres au diplômé $(\mathrm{bac}+5)$ :

- langues étrangères ;

- management, gestion, gestion de projet, hygiène et sécurité, environnement, (règlement REACH sur l'enregistrement, l'évaluation, l'autorisation et les restrictions des substances chimiques...) ;

- analyse du cycle de vie complet d'un produit ou d'une machine pour répondre aux exigences liées au développement durable, etc.

Ces demandes accrues ne se sont pas accompagnées d'une augmentation de la durée de formation. Bien au contraire, le nombre d'heures d'enseignement proprement dit a diminué. Il a fallu rajouter des stages de plus en plus longs afin de rendre les jeunes diplômés opérationnels plus rapidement. 
En résumé, l'étudiant en mécanique aborde plus de choses qu'il y a vingt ans, mais les fondements scientifiques sont vus plus rapidement. L'entrée dans l'enseignement supérieur est moins sélective. Les exigences dans le cœur de métier sont plus larges, ainsi que dans des domaines périphériques. La formation est toujours du même nombre d'années, mais réduite en heures effectives d'enseignement. Notre système de formation est donc malade!

Contrairement à la recherche, les rencontres consacrées à la pédagogie ne sont pas nombreuses, ce qui limite les échanges entre enseignants. On peut citer le CETSIS, Colloque sur l'enseignement des technologies et des sciences de l'information et des systèmes, qui concerne la communauté en sciences pour l'ingénieur au sens large (EEA, mécanique, optique, physique...) ou encore de manière plus spécialisée la session «Formation et pédagogie » du Congrès français de mécanique (CFM). On peut constater que si initialement les nouvelles technologies de l'information et de la communication (NTIC) étaient à l'honneur au CFM, elles ont fait de la place depuis environ cinq ans aux exposés sur des sujets visant à augmenter l'intérêt et la motivation des étudiants (passage de "ceintures " ${ }^{9}$, vote des étudiants en cours pour répondre à une question par télévoteurs ${ }^{10} \ldots$ ) et bien sûr la mise en place de la formation par projets et par compétences ou méthode de classe inversée. On peut également mentionner l'arrivée, qui n'est peut-être qu'une mode, des massive open online course, qui fera nécessairement bouger les choses. Néanmoins, même si toutes ces initiatives, visant à motiver davantage l'étudiant et à le rendre acteur de sa formation, sont louables et indispensables, elles ne sont que des médicaments de confort, des palliatifs partiels, qui ne peuvent pas guérir la maladie dont est atteint notre système de formation.

\subsubsection{Propositions}

Il faut agir au plus vite dans l'enseignement supérieur pour les diplômés de demain, mais également au niveau de l'enseignement primaire et secondaire pour ceux d'après-demain. Les actions à entreprendre doivent non seulement soulager les principaux maux de notre système, mais également les guérir.

Le niveau des lycéens français diminue par rapport à ceux des autres pays de l'OCDE. Il est donc nécessaire d'amorcer au plus vite un changement avec un retour vers les bases fondatrices. Dans l'enseignement primaire et secondaire, il faut redéfinir les fondamentaux nécessaires pour la future vie professionnelle de l'enfant, en mathématiques, en physique et en français, en plus, bien sûr, d'en faire un citoyen. Ces fondamentaux doivent être prioritaires. Par conséquent, il est indispensable de laisser du temps à l'élève pour les assimiler. Il faut éviter la dispersion : est-ce bien utile que des élèves de terminale scientifique voient la mécanique quantique, sujet traité préalablement dans le supérieur ? Les mêmes questionnements

9 A. Pelat, J.-M. Génevaux, S. Gougeon, J.-P. Dalmont, N. Joly, « Leur remonter les bretelles avant l'examen ou leur faire passer des ceintures ?... De quoi donner des boutons! ", Congrès français de mécanique, Bordeaux, 26-30 août 2013.

${ }^{10}$ N. Régnier, «Systèmes de réponse instantanée pour une pédagogie active », Congrès français de mécanique, Bordeaux, 26-30 août 2013. 
se posent en mathématiques avec la suppression de certains fondements remplacés par des notions "dont l'intitulé fait plutôt penser à des recherches avancées de spécialistes ${ }^{11}$. La dispersion et la non-compréhension de certains fondements par manque de temps est peut-être une des causes de la désaffection des sciences par les élèves, celles-ci étant réputées dures. Effectivement, les sciences font appel à des notions complexes, mais compréhensibles si on laisse le temps à l'élève de les assimiler, alors qu'elles deviennent rebutantes si elles ne sont pas comprises.

Dans l'enseignement supérieur, nous devons former au plus près des besoins industriels et donc au mieux les mécaniciens de demain. Comme cela a été argumenté plus haut, les exigences dans le cœur de métier sont plus nombreuses, ainsi que dans d'autres domaines liés au métier de l'ingénieur au sens large. En revanche, la formation est toujours de la même durée, voire réduite en heures effectives d'enseignement. Deux moyens d'action sont possibles : réaménager les compétences demandées à durée de formation constante, ou augmenter la durée de formation à compétences demandées constantes.

Les propositions ci-dessous peuvent toutes coexister. Elles sont illustrées au niveau bac +5 ; néanmoins, elles peuvent aisément être adaptées à d'autres niveaux. La première proposition consiste en un réaménagement des compétences à durée de formation constante et les deux suivantes impliquent une augmentation de la durée de formation.

- À durée de formation constante, et par conséquent à volume de formation équivalent, les ambitions pédagogiques doivent être réaménagées. À l'opposé du référentiel actuel unique définissant l'ingénieur/le master, redéfinissons-le en fonction du métier ciblé. Cependant, aucune concession ne doit être faite sur le socle des sciences fondamentales dont la mécanique constitue une large part : le diplômé doit les maîtriser. Les réaménagements doivent être opérés dans certaines disciplines bien ciblées. L'ingénieur mécanicien en entreprise est, en fonction de ses aspirations, de sa personnalité et de ses aptitudes, un scientifique expert ou un scientifique manager. Sa formation peut donc être déclinée selon ces deux orientations. Le scientifique expert doit être très performant en science et/ou technologie, mais peut avoir une formation allégée en sciences humaines et sociales (management, gestion de projet, qualité...), ce qui représente aujourd'hui environ $20 \%$ de la formation d'un ingénieur. Le scientifique manager peut être un mécanicien un peu moins performant sur certains aspects. Il ne lui est pas demandé de maîtriser de logiciel de $\mathrm{CAO}$, mais la conception mécanique doit lui être familière. Il n'est pas un expert en calcul numérique, ne connaît pas forcément les codes de calcul les plus connus, mais doit pouvoir faire un calcul de résistance des matériaux «à la main ». En revanche, sa formation en sciences humaines et sociales doit être solide.

Cette solution permet de recentrer les compétences demandées sur le métier du mécanicien en entreprise, sans handicaper le futur ingénieur dans sa carrière. En effet, l'étudiant dispose de plus de temps pour assimiler les

${ }^{11}$ http://www-fourier.ujf-grenoble.fr/ demailly/manuscripts/communique_math_TS_2011.pdf 
notions qui lui sont enseignées. Il est à souligner qu'aucun moyen humain et financier supplémentaire n'est nécessaire. Pour qu'au cours de sa vie professionnelle le scientifique expert puisse devenir scientifique manager, ou inversement, il faut que l'accès à la formation continue ou par alternance soit grandement facilité. Une politique dynamique de validation des acquis de l'expérience doit être menée en concertation avec l'industrie.

- Une autre solution est que la formation au niveau bac +5 (master ou ingénieur) comporte une année supplémentaire réalisée en alternance. Toutes les compétences actuellement demandées sont validées, mais une partie de la formation est alors externalisée dans les entreprises. En effet, il est envisageable de confier certains modules de formation aux entreprises partenaires, la validation étant effectuée par l'établissement délivrant le diplôme : management, gestion de projet, qualité, etc. La formation gagnerait certainement en efficacité, les entreprises étant en général plus performantes que les enseignants-chercheurs dans la plupart de ces domaines. Il est alors nécessaire de définir préalablement l'attente que l'établissement de formation a de l'entreprise.

- La troisième solution consiste à allonger la durée de formation pour permettre d'atteindre les niveaux de formation requis aujourd'hui en termes de compétences. Cela est envisageable dans le système européen LMD (licence, maîtrise, doctorat) qui est basé sur la capitalisation de compétences via des unités d'enseignement dont la valeur est comptée en ECTS (European Credits Transfer System). Un étudiant peut assimiler plus à son rythme certaines notions difficiles et réussir à valider les crédits ECTS nécessaires pour l'obtention du diplôme, sans que cela soit synonyme pour autant de redoublement. L'étudiant peut préparer en un semestre un nombre de crédits ECTS inférieur à 30 (nombre de crédits préparés dans le système actuel en un semestre).

Ces solutions sont toutes envisageables à surcoût réduit, voire nul. Certaines présentent l'avantage d'impliquer davantage les entreprises. Cependant, elles supposent toutes que le référentiel des formations d'ingénieur ou de master $(\mathrm{bac}+5)$ soit flexible pour s'adapter à la situation actuelle décrite dans la première partie de ce chapitre. L'enseignement supérieur a la capacité et la réactivité suffisante pour agir vite, mais laissons-lui la possibilité :

- de former des scientifiques experts ou des scientifiques managers en cinq ans. Pour les deux types de scientifiques, experts ou managers, cela suppose que l'évolution de salaire soit identique, comme cela est pratiqué dans certaines entreprises, fleurons de l'industrie française ;

- d'allonger la formation d'un an en associant des entreprises pour des compétences « métier » complémentaires ;

- d'adapter la durée de la formation au rythme de l'étudiant.

Faisons de l'enseignement des fondamentaux une priorité ! Des solutions existent ; donnons-nous collectivement la liberté de les mettre en œuvre ! 
